\title{
Research Paper \\ Effect of Kinesio Taping on Ankle Joint Kinematics During Landing on Stable and Unstable Surfaces in Ankle Sprain and Health Persons
}

\author{
Mohammad Baghbani ${ }^{1}$ (1), "Mohammadtaghi Amiri-Khorasani ${ }^{1}$ (1), Abdolhamid Daneshjoo ${ }^{2}$ (1)
}

1. Department of Sport Biomechanics, Faculty of Physical Education and Sport Sciences, Shahid Bahonar University of Kerman, Kerman, Iran. 2. Department of Pathology and Corrective Exercises, Faculty of Physical Education and Sport Sciences, Shahid Bahonar University of Kerman, Kerman, Iran.

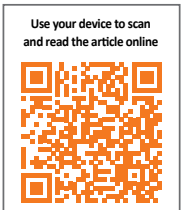

Citation Baghbani M, Amiri-Khorasani M, Daneshjoo A. [Effect of kinesio Taping on Ankle Joint Kinematics During Landing on Stable and Instable Surfaces in Ankle Sprain or Health Persons (Persian)]. Scientific Journal of Rehabilitation Medicine. 2021; 10(3):522-531. https://doi.org/10.32598/sjrm.10.3.12

doi https://doi.org/10.32598/sjrm.10.3.12

Received: 10 May 2021 Accepted: 30 Jun 2021 Available Online: 23 Jul 2021

Keywords:

Landing, Kinematics, Kinesio tape, Ankle joint, Ankle sprain

\begin{abstract}
Background and Aims Landing is a typical sports motion that can create impact force 2-12 times of body weight, and finally, it's one of the main reasons for non-contact injuries in ankle ligaments. Specialized. The usual effects of Kinesio tape include increasing proprioception, health direction of joints, reducing pain, and raising pressure on nervous tissue. The study aimed to investigate the effect of Kinesio taping on ankle joint kinematics during landing on stiff and soft surfaces in ankle sprain and healthy persons. Methods The method of the present study was quasi-experimental with a two-group design in control groups (without ankle sprain) and experimental (with an ankle sprain). A total of 30 male students of the Shahid Bahonar University of Kerman were purposefully and accessibly selected and divided into two groups with (15 students) and without ankle sprains ( 15 students). Then, they performed both landing operations on stable and unstable surfaces, with and without Kinesio tape. Maximum dorsi and plantar flexion, supination, pronation and maximum ankle angular velocity parameters were recorded by a three-dimensional motion analysis system. Statistical analysis was performed using independent t-test and repeated measures analysis of variance at the significant level of 0.05 .

Results There was no significant reduction in plantar flexion of the ankle in healthy and twisted individuals while landing on stable and unstable surfaces with and without Kinesio tape $(P \leq 0.07)$, but there was a significant reduction in the dorsiflexion in both groups $(\mathrm{P} \leq 0.001)$. On the other hand, there was no significant decrease in pronation $(P \leq 0.66)$, but there was a significant decrease in foot supination $(P \leq 0.001)$.

Conclusion Generally, Kinesio tape in recovery ankle movement is offered to persons for ankle sprain. Thus recommendation landing exercises fare with more flexion angle and less knee joint valgus and more dorsiflexion angle at ankle joint and preferable on the unstable surfaces.
\end{abstract}

\section{Extended Abstract}

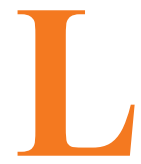

\section{Introduction}

anding is one of the motions that can create an impact force 2 to 12 times the body's weight, which is often associated with the mechanisms of lower limb injury. This mechanical shock must be adjusted through the musculoskeletal system. According to the literature, $58 \%$ of all female basketball players' injuries occur following landing due to a jump; one of these common anomalies in the ankle joint is inward and outward rotation of the toe. Natural misalignment of the knee and ankle joint can cause ab-

\section{${ }^{*}$ Corresponding Author:}

Mohammadtaghi Amiri-Khorasani, PhD.

Address: Department of Sport Biomechanics, Faculty of Physical Education and Sport Sciences, Shahid Bahonar University of Kerman, Kerman, Iran Tel: +98 (34) 33257623

E-Mail: amirikhorasani@uk.ac.ir 
normalities such as osteoarthritis, wear and tear, and ankle sprains. As the surface stiffness increases, so does the foot stiffness, and as the surface stiffness decreases, the foot stiffness decreases.

One of the methods used to reduce ankle sprains is the Kinesio tape, one of the most common and practical methods to prevent this type of injury. The Kinesio tape does not impede normal movement in the plantar and dorsiflexion range of motion. It aims to support and stabilize the ankle by inhibiting inactive synergists or antagonists, facilitating impaired motor synergists, increasing proprioception, improving joint alignment, reducing pain, and relieving pressure on irritated nerve tissues. The Kinesio tape is suggested for prevention by increasing depth, improving joint alignment, reducing pain, and relieving pressure on nerve tissue.

Therefore, previous studies have separately evaluated the effect of Kinesio tape on ankle sprains. However, no study was found that simultaneously measured the effect of Kinesio tape on disturbed surfaces on ankle kinematics using precision motor analysis tools. Therefore, this study aimed to investigate the effect of Kinesio tape on ankle kinematics during landing on stable and unstable surfaces in individuals with and without ankle sprains.

\section{Methods}

This study's method was quasi-experimental with a twogroup design in control groups (without ankle sprain) and experimental (with an ankle sprain). A total of 30 male students of the Shahid Bahonar University of Kerman were purposefully and accessibly selected and divided into two groups with (15 students) and without ankle sprains (15 students).

The selection of the subjects with ankle sprains was based on the standard method mentioned in previous research. The issues had criteria such as experiencing moderate to severe ankle sprains at least once in the past year, feeling the empty joint inside, and chronic ankle instability in only one foot. The diagnosis of ankle sprain was also made by MRI image and doctor's opinion. On the test day, patio reflex markers were first affixed to the fifth and first metatarsals, heel, talocalcaneal joint, the posterior and middle part of the heel joint, external ankle, thin outer condyle. Then the warm-up program was performed, which included 4 minutes of soft running and then stretching and relaxing movements of the lower limb muscles. Then, they performed both landing operations on stable and unstable surfaces, with and without Kinesio tape.
In this study, typing was performed by a physiotherapist, and a $5 \mathrm{~cm}$ wide Kinesio tape was used, which can be stretched by $40 \%$ and is waterproof and anti-allergic. This study's dependent variables were maximum dorsiflexion, maximum plantar flexion, maximum supination, maximum pronation, and maximum angular velocity. Therefore, the study stage was in the landing motion from foot contact with the ground until the ankle joint reached the maximum dorsiflexion. In this stage, the measured variables at foot contact with the ground up to the maximum dorsiflexion were examined.

The kinematic variables of the landing were recorded during the period from the beginning of the movement to the continuation of the movement after landing, and a three-dimensional system of motion analysis recorded the required factors. Statistical analysis of data was performed using an independent t-test and repeated measures analysis of variance at a significant level of 0.05 .

\section{Results}

According to this study, people with ankle sprains have less flexion in the ankle joint than healthy people when the dorsal landing $(\mathrm{P}=0.001)$. According to the repeated combined analysis statistical test, the effect of Kinesio tape on the amount of plantar flexion of the ankle during landing on a stable and unstable surface between healthy individuals with ankle sprains was not significant $(\mathrm{P}=0.148)$. There was also a significant decrease in the rate of ankle supination in people with and without ankle sprains $(\mathrm{P}=0.001)$. The data did not show a significant reduction in ankle pronation in healthy and ankle sprain individuals $(\mathrm{P}=0.205)$. There was a significant decrease in the angular velocity of the ankle in the two groups with and without an ankle sprain $(\mathrm{P}=0.001)$.

\section{Discussion and Conclusion}

This study results showed people with ankle sprains land on the supine angle, and the speed of the ankle angles is less than healthy people when landing. Due to the lower angular velocity of healthy individuals than those with ankle sprains, it is expected that the risk of ankle injury is higher in the ankle sprained group. It should be noted that the unstable surface also increases the angular velocity, which in turn creates a greater angular slope. There was also a significant difference in the parameter of ankle dorsiflexion in healthy and ankle sprained individuals while landing on a stable and unstable surface with and without Kinesio tape. 
This reduction, which occurred due to the use of Kinesio tape, seeks to increase greater stability of the ankle due to increased motor control of the nervous system and a reduction in disturbances in the afferent messages of the ankle joint. Since reducing the dorsiflexion angle is known as a risk factor for ankle ligament injury, proper training and modification of the landing pattern is necessary to reduce the loads applied to the external ankle compartment. The use of Kinesio tape can also help prevent injury.

\section{Ethical Considerations}

\section{Compliance with ethical guidelines}

This study was approved by the ethics committee from the Shahid Bahonar University of Kerman (IR. UK.REC.1400.004). Also, all ethical principles are considered in this article. The participants were informed about the purpose of the research and its implementation stages. They were also assured about the confidentiality of their information and were free to leave the study whenever they wished, and if desired, the research results would be available to them.

\section{Funding}

This study was extracted from the MSc. thesis of first author at Department of Sports Biomechanics of Sports Science Faculty of Shahid Bahonar University of Kerman.

Authors' contributions

Authors contributed equally in preparing this article.

\section{Conflict of interest}

The authors declared no conflict of interest. 


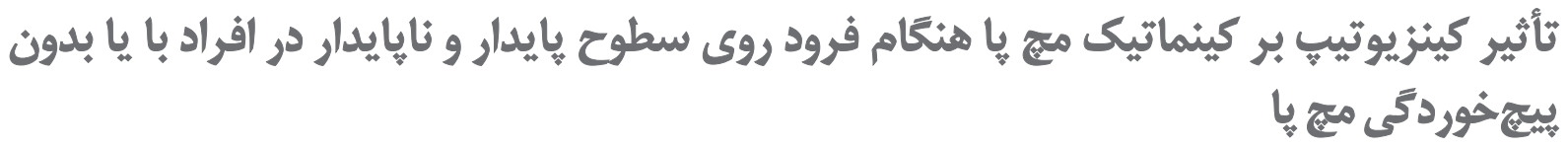

محمد باغبانى' (1). "محمدتقى اميرى خراسانى' (1)، عبدالحميد دانشجو' (1)

ا. كروه بيومكانيك ورزشى، دانشكده تربيتبدنى و علوم ورزشى، دانشكاه شهيد باهنر كرمان، كرمان، ايران.

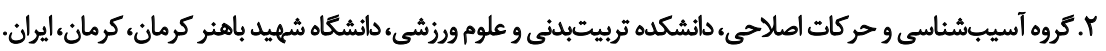

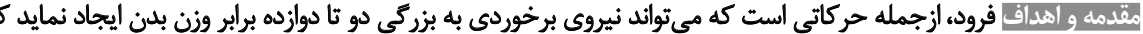

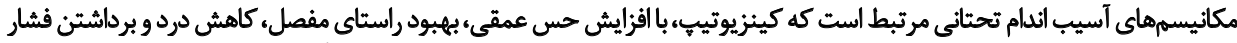

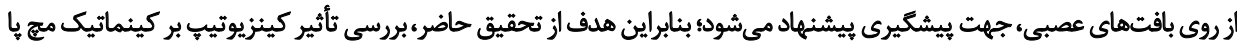

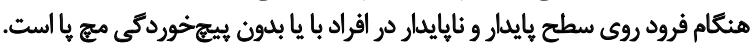

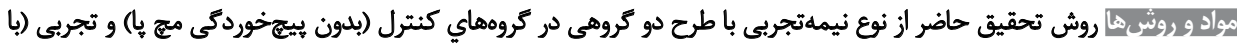

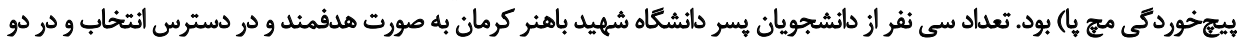

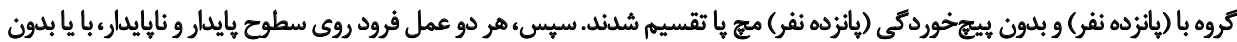

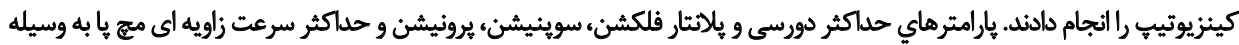

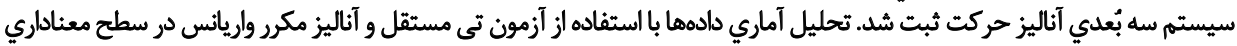

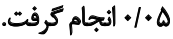

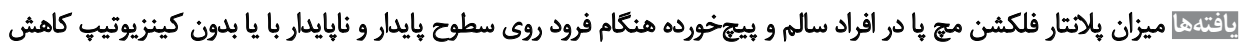

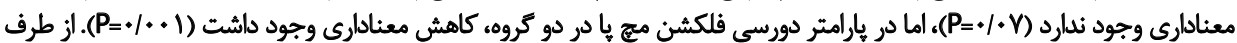

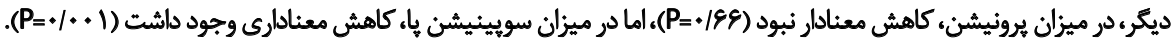

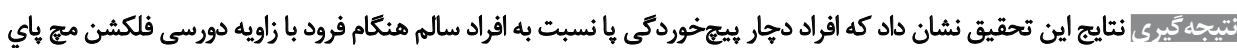

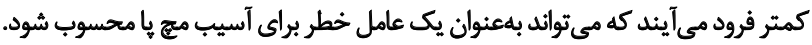

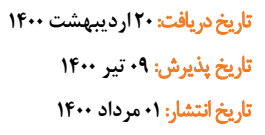

:

فرود، كينماتيك، فرود، مفصل مجيج ياه

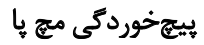

شيوع را دارد. يكى از عوامل اين آسيب، فرود' است كه ازجمله

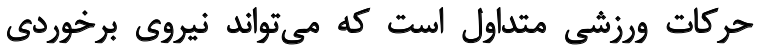

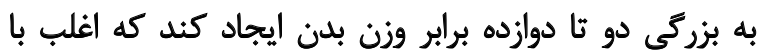

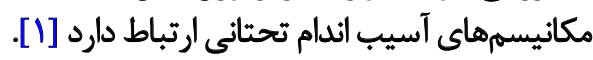

يكى از اهداف مهم تمرينات بدنى، كسب و نتُهداري آمادتي

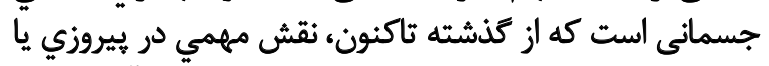

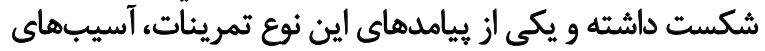

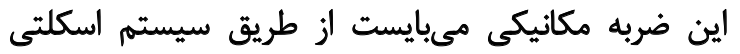

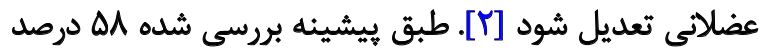

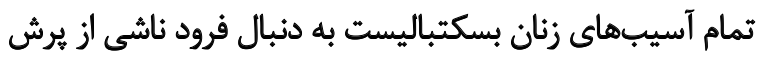

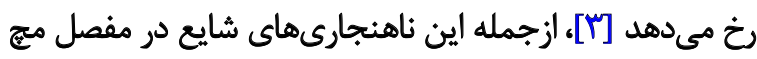

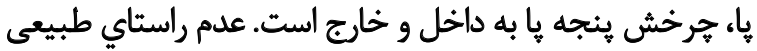

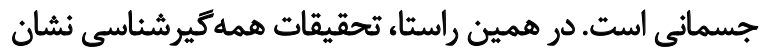

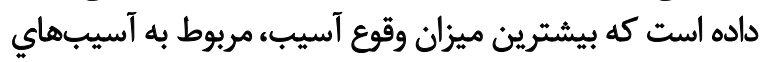

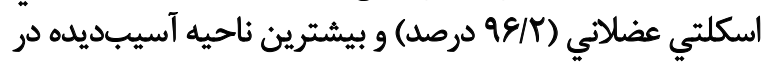

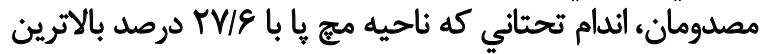

1. Landing 


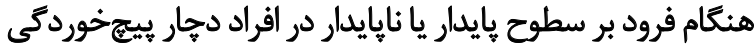
ميج يا و افراد سالم را در اين تحقيق بروسي كنئد.

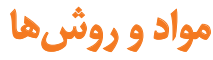

روش تحقيق حاضر از نوع نيمهتجربى با طرح دو گروهى بروبى

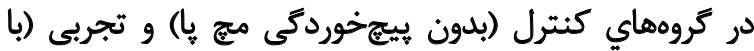

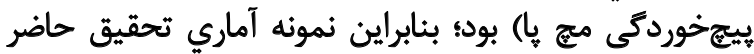

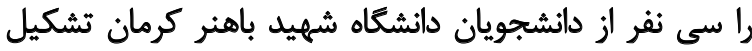

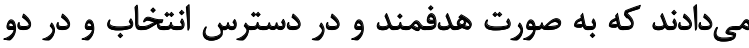

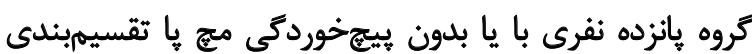

شدند (جدول شماره ()).

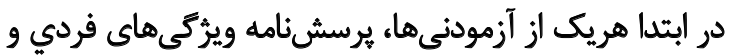

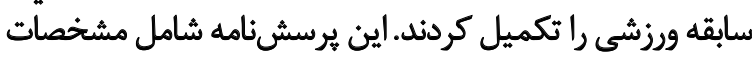

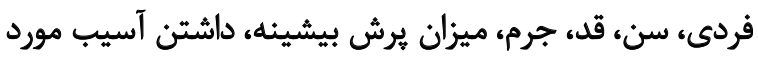

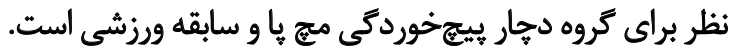

شيوه انتخاب افراد دجار يبيجخوردكى ميج يا بر اساس شيوه

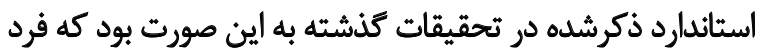

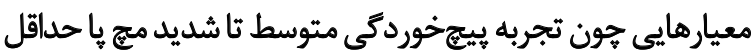

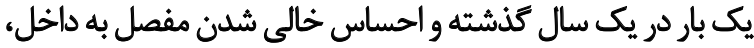

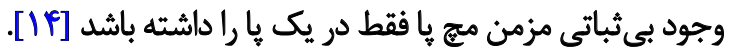

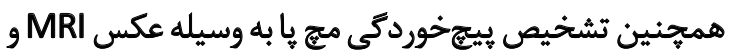

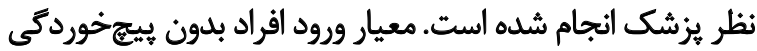

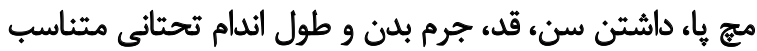

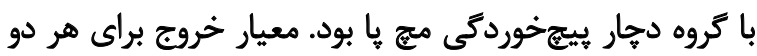

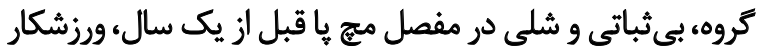

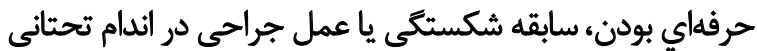

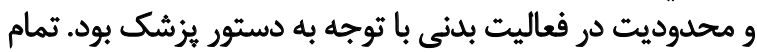

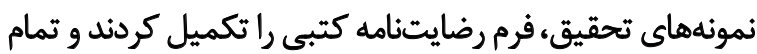

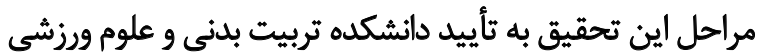
دانشكاه شيديد باهنر كرمان رسيده است.

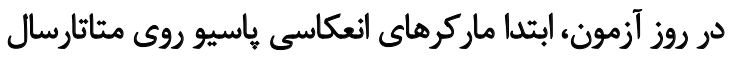

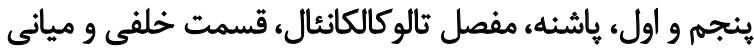

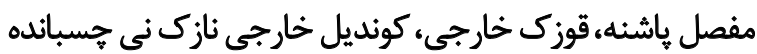

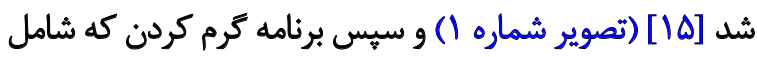

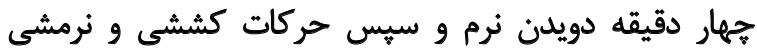

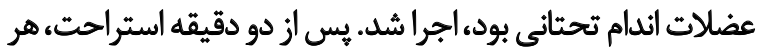

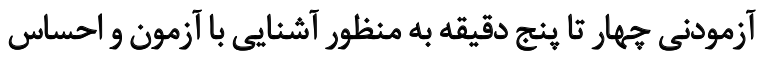

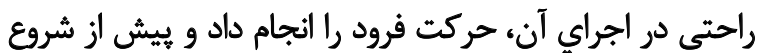

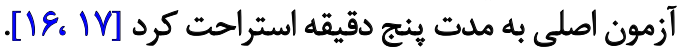

هر آزمودنى روى يك سطح، سه برش با فاصله يك تا سه

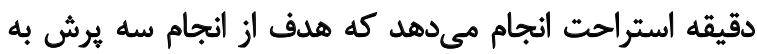

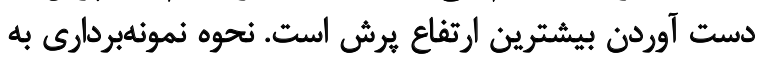

در مفصل زانو و ميج يا موجب بروز ناهنجارىهايى هون آرتروز،

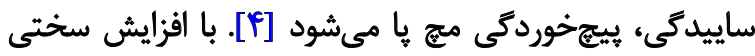

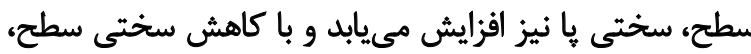

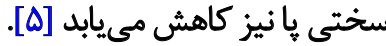

هنكام فرود متعاقب يرش عمودي به محض تماس، نيروهاي

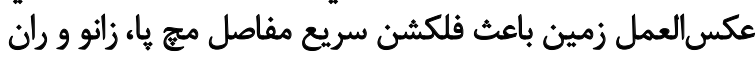

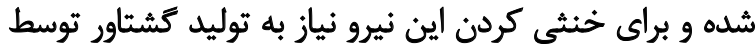

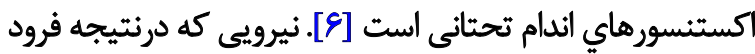

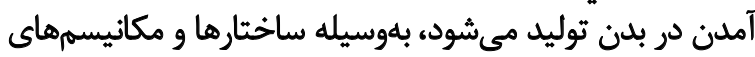

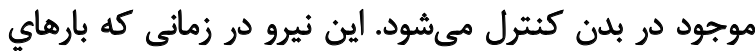

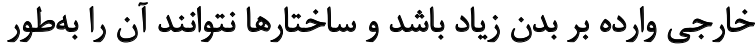

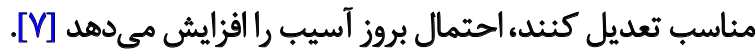

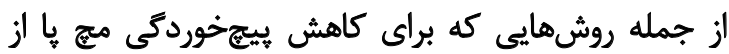

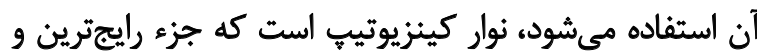

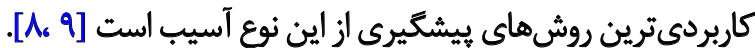

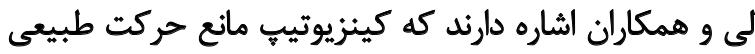

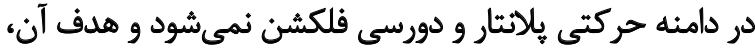

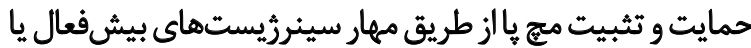

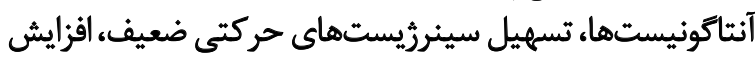

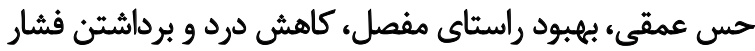

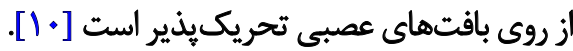

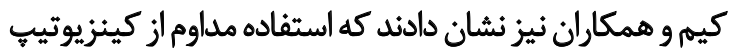

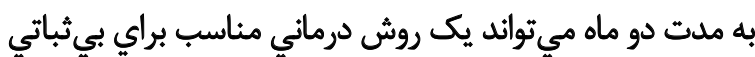

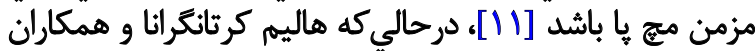

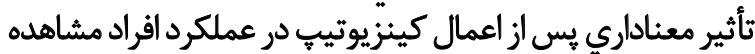

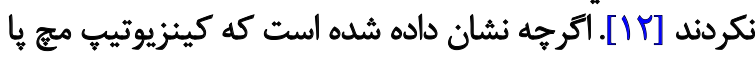

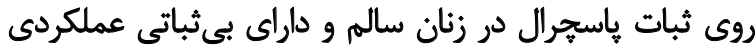

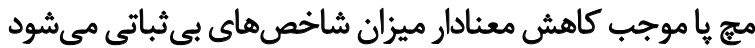

ازز طرف ديكر، صفرىبك و همكاران كزارش كردند كه استفاده

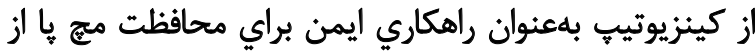

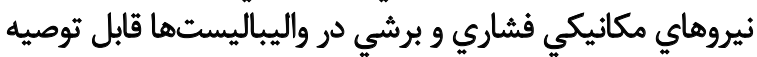

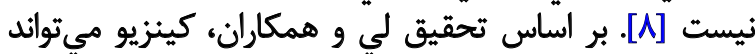

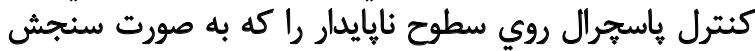

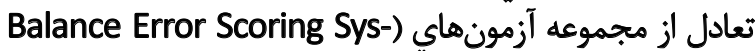

tem (BESS بنابراين با بررسىهاى صورت كرفته، تحقيقات كذشتيه به

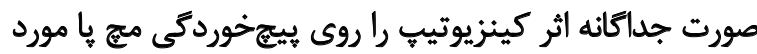

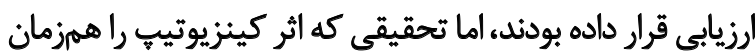
روى سطوح داراى خش بر كينماتيك مج يج با با استفاده از ابزار

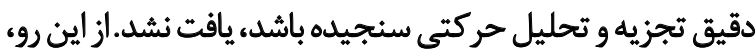

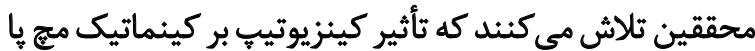


جدول ا.ويزگكىهاى جمعيتشناختى آزمودنىها

\begin{tabular}{|c|c|c|c|}
\hline $\mathbf{P}$ & مياتغين||نحراف معيار & تمروه & شاخص \\
\hline ./199 & $\begin{array}{l}r / \& \Lambda \pm Y / q . \\
r T / T \Delta \pm Y / . r\end{array}$ & سيتخورده & سن (سال) \\
\hline r & 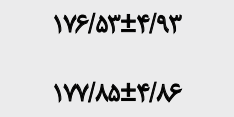 & سيجن نورده & قداند (سانتىثت) \\
\hline - /A9F & $\begin{array}{l}v \in / . r \pm 1 \cdot / r \cdot \\
v \Delta / q \Psi \pm q / T^{\prime}\end{array}$ & بيتخورده & جرم بلن (كيلوكرم) \\
\hline
\end{tabular}

تقريباً • + أ درصد حداكثر طولش كشيده و در زير برجستكى تيبا

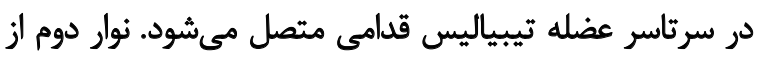

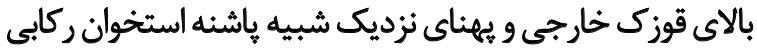

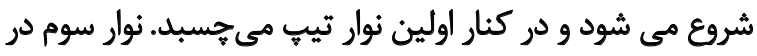

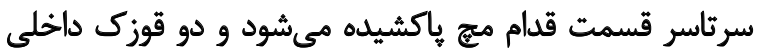

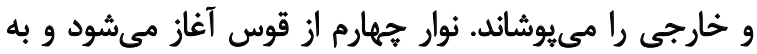

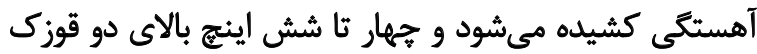

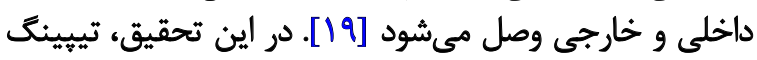

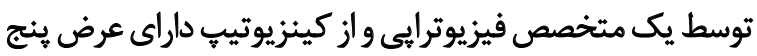

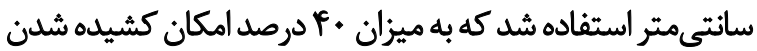

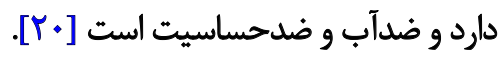

\section{جمعآورى دادهاي كينماتيكي}

حداكثر دورسى فلكشن، حداكثر بلانتار فلكشن، حداكثر

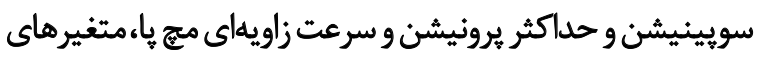

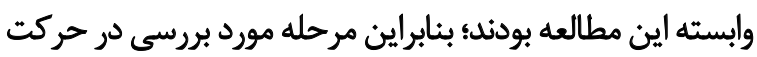

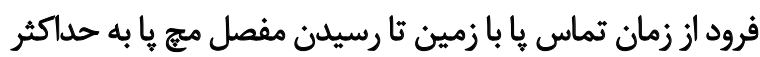

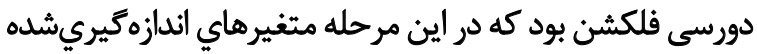

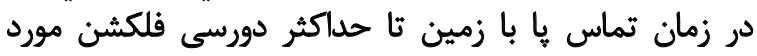

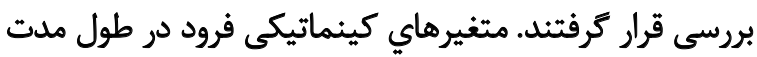

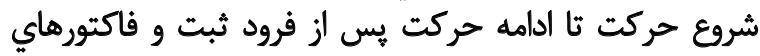

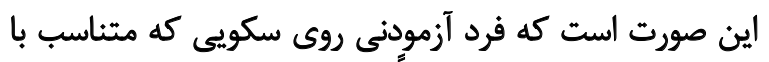

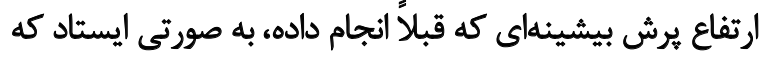

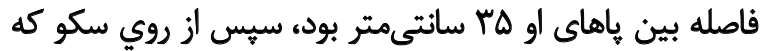

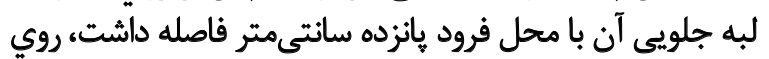

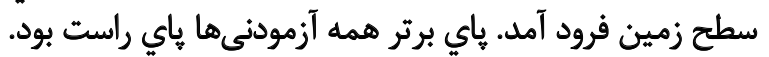

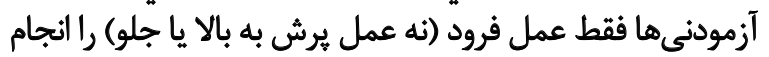

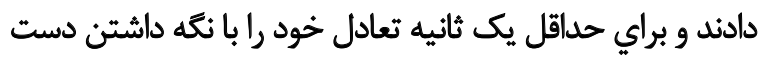

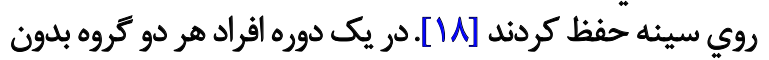

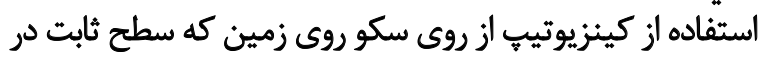

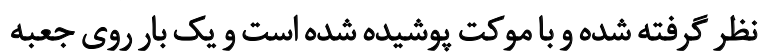

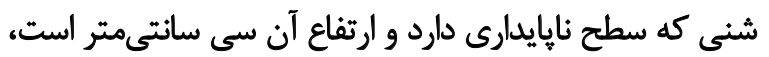

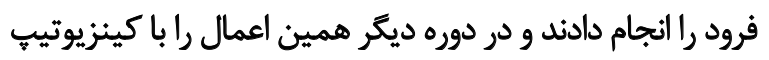

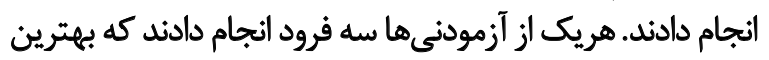
فرود ثبت مىشد (تصوير شماره اند).

\section{نحوه بستن كيئزيوثيبي}

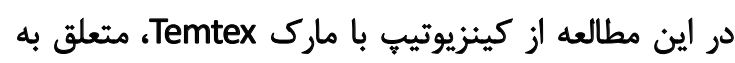
شركت تواتك، ساخت كشور كره استفاده شد كه در مر مسابقات

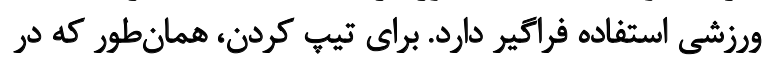

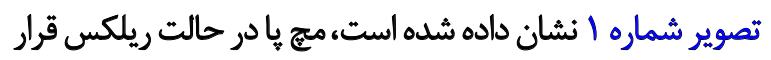

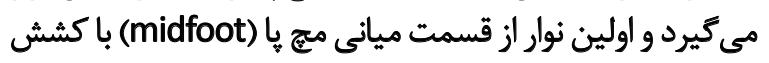

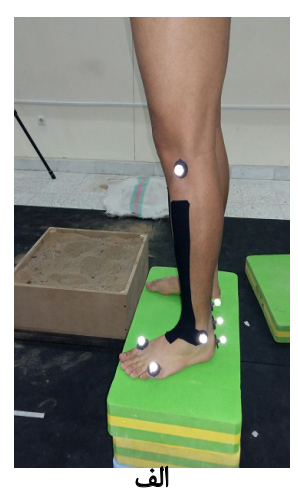

تصوير ا. محل ماركرهاى يسيو و نحوه فرود روى سطح بايدار (الف) و نايايدار (ب) 
جدول r. ميانكين و انحراف استاندارد متغيرهاى تحقيق در حالات مختلف (هر كروه شامل افراد سالم و بيجخورده است)

\begin{tabular}{|c|c|c|c|c|}
\hline \multicolumn{4}{|c|}{ مياتكين||نحراف معيار } & \multirow[t]{2}{*}{ متغيرها } \\
\hline كينزيوتيب نإيايدار & كينزيوتيب بايدار & نإيايدار & ن إيدار & \\
\hline $10 / 1 \pm \pi / \pi$ & $\mid Q / / \pm N \Delta$ & $\mid \omega / \backslash \pm V / \pi$ & $18 / 1 \pm \pi / \Delta$ & دورسى فلكشن (درجه) \\
\hline$T \Psi / T \pm E / T$ & $r \Delta / Y \pm S / P$ & $\Gamma+T \pm T / \%$ & $r \Delta / Y \pm V / \Delta$ & ياهنتار فلكشن (درجه) \\
\hline$T / \Psi \pm \Delta / q$ & $n / r \pm n$ & $r / T \pm V q$ & $r \in / r \pm V / r$ & سويئيشن (درجه) \\
\hline$V / T \pm \mathscr{R}$ & $N T \pm \Delta / \Delta$ & $V / T \pm \Delta / r$ & $N Y \pm T / S$ & يروتيشن (درجه) \\
\hline$\varepsilon \cdot V / / r / \pm 1 / q$ & $9 \cdot \Delta / 111 \pm \pm 1 / 4$ & AN"/IFE $\pm \& T / q$ & $1.95 / 11+ \pm r / 1$ & عت زاويهاي (درجه بر ثاثيه) \\
\hline
\end{tabular}

خدى دو در جدول Mauchly's Test معنادار نبود، از دادههاى

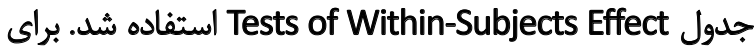

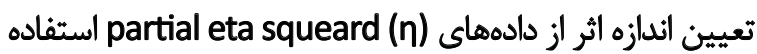

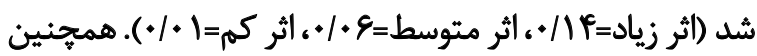

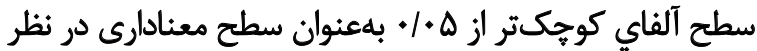
كرفته شد.

Liأits

در جدول شماره Y ميانكين و انحراف استاندارد متغيرهاى

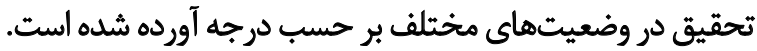

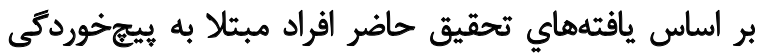

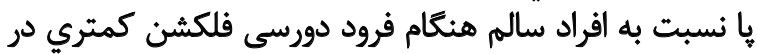

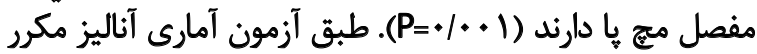

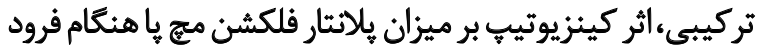

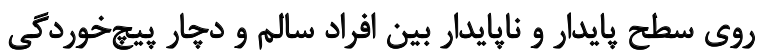

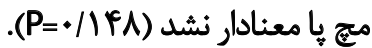

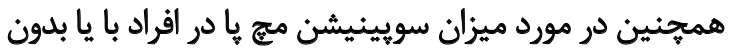

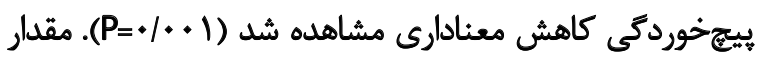

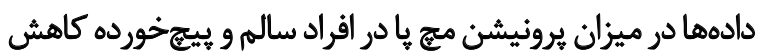

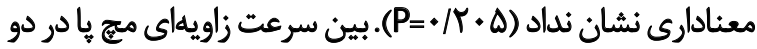

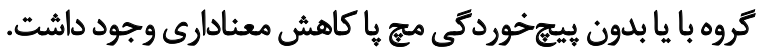

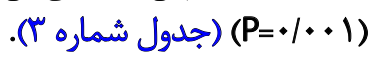

$\leftrightarrow$

بلهوركلى نتايج تحقيق حاضر نشان داد كه افراد دجار

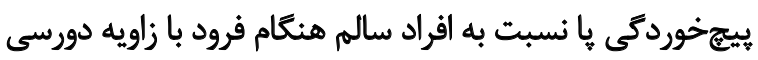

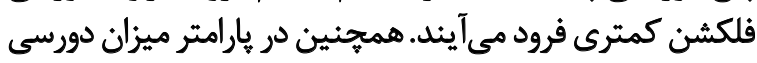

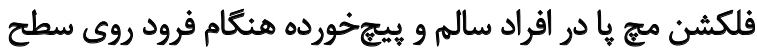

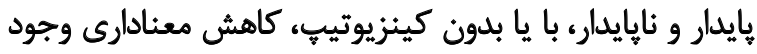

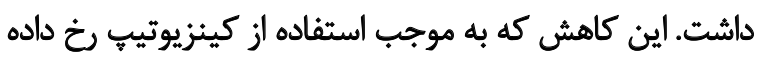
است، به دنبال افزايش ثبات بيشتر مج با به دليل افئ افزايش كنترل
خواستهشده تجزيه و تحليل شد [ [1 ،9]] براي به دست آوردن

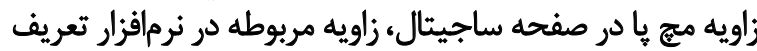

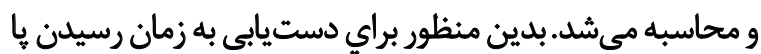

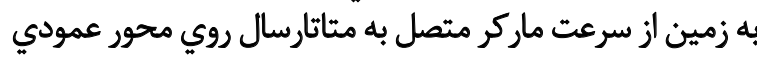

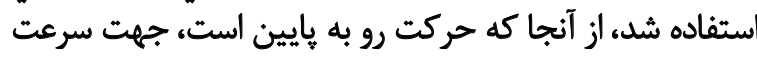

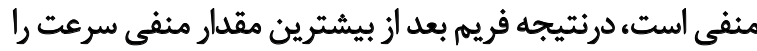

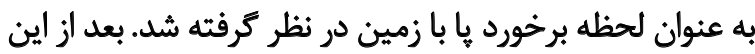

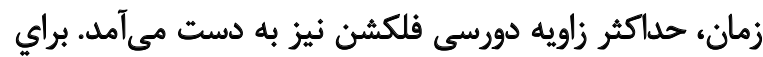

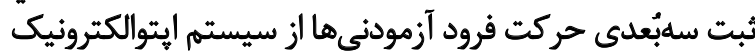
سهبُعدى Motion Analysis با شش دوريت فرونين استفاده شد. دادههاي ثبتشده، به وسيله نرمافزار

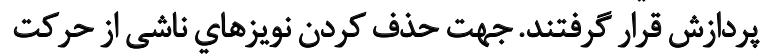

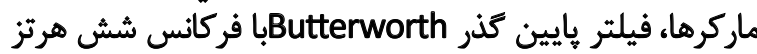

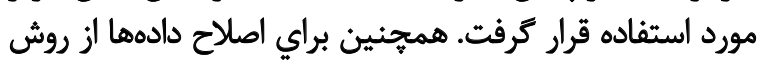
Rectify و Smoothing

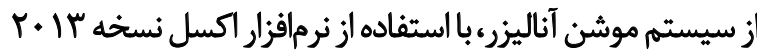

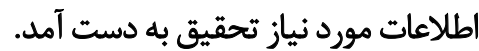

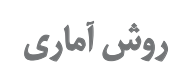

در اين تحقيق براى آناليز دادهها از نرمافزار SPSS مدل

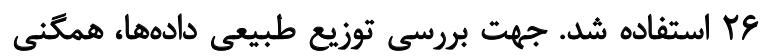

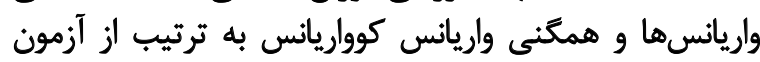

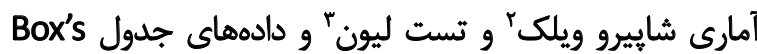

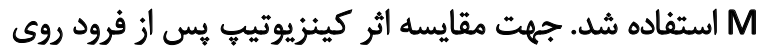

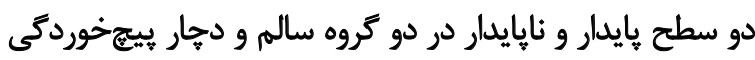

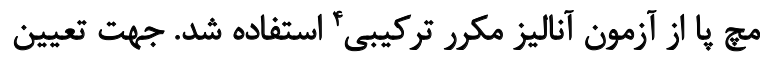

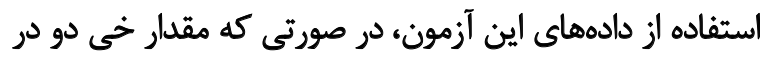

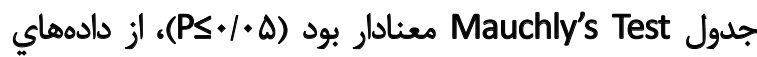
جدول Multivariate Test استفاده شد. در صورتى كه مقدار

\section{Shapiro Test}

3. Lion Test

4. ANOVA With Repeated Measure7 Chi-Square 


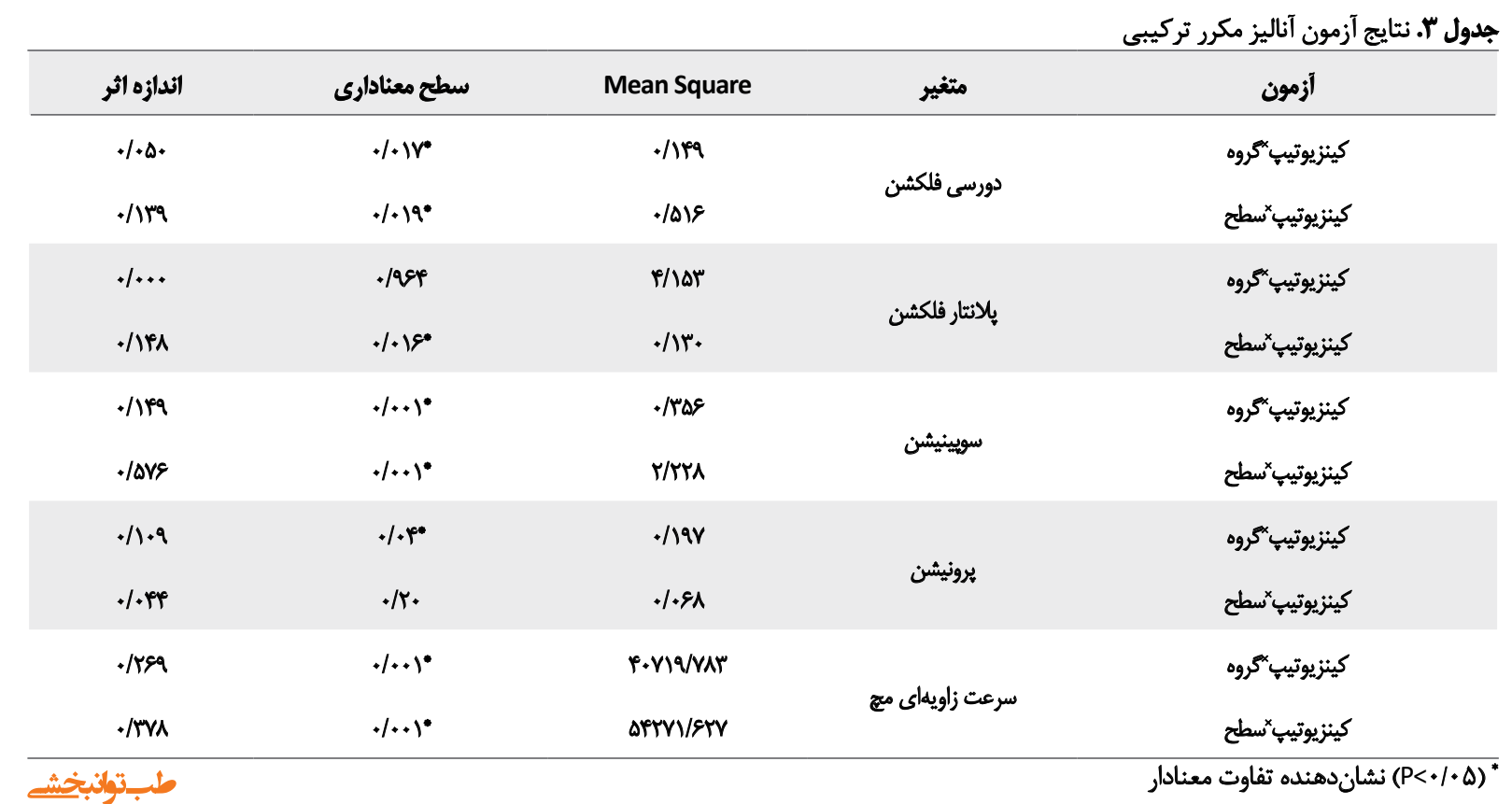

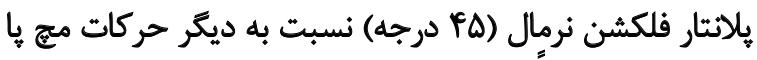

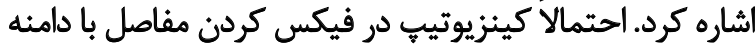
حركتى زياد اثر مثبت كمترى دارد.

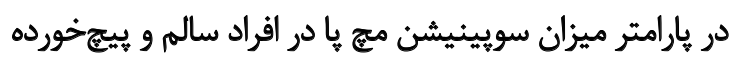

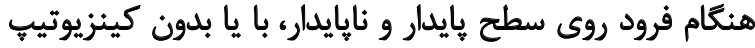

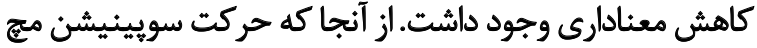

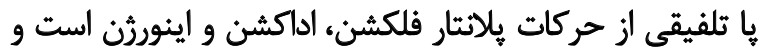

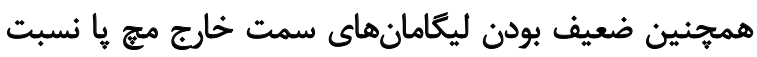

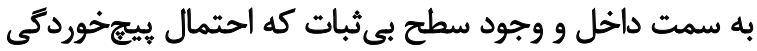

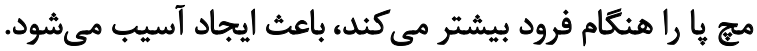

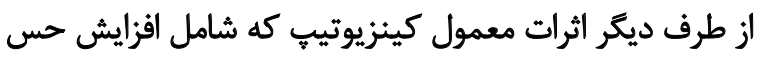

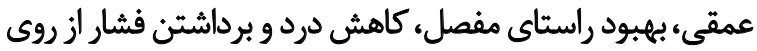

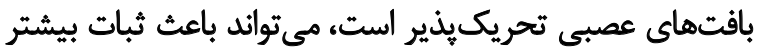

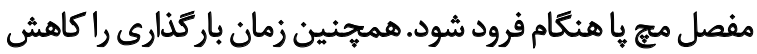

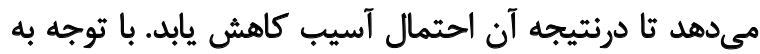

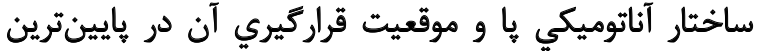

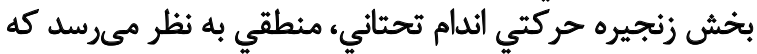

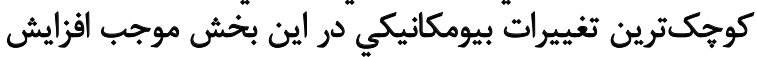

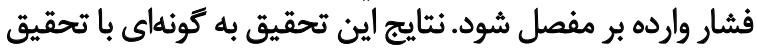

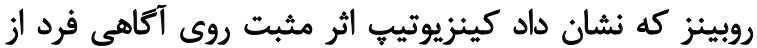

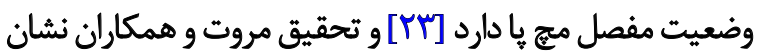

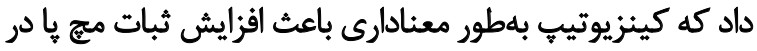

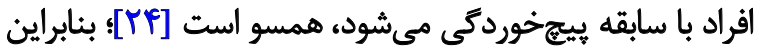

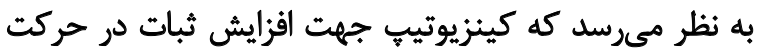

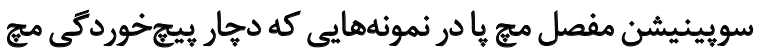

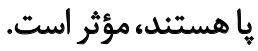

حركتى سيستم عصبى و همجنين كاهش اختلال در بيامهاى

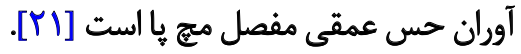

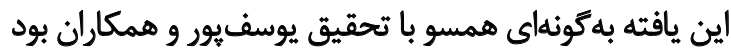

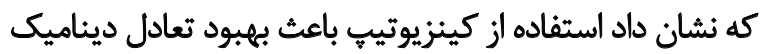

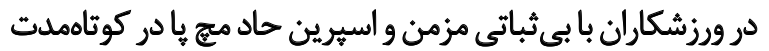

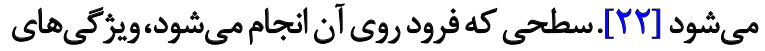

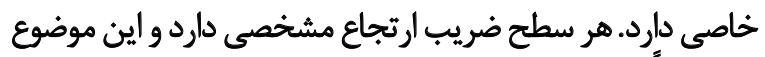

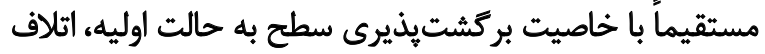

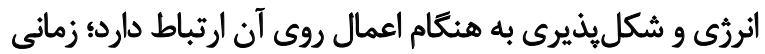

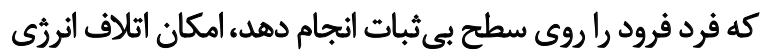
وخطر آسيب وجود دارد.

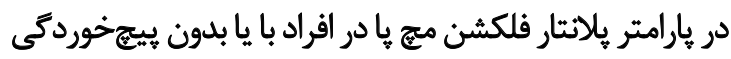

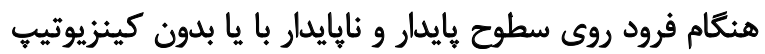

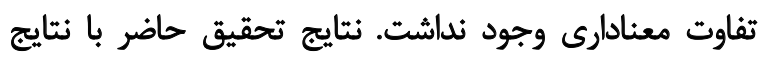

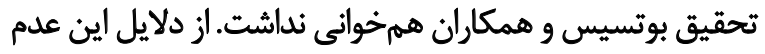

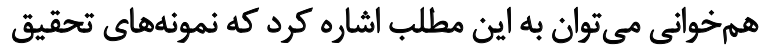

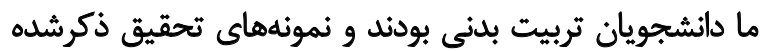
ورزشكاران باله بودند.

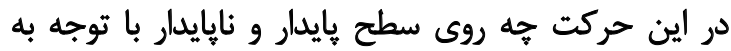

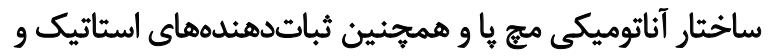

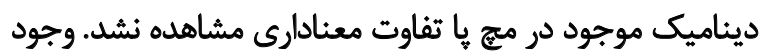

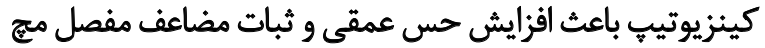

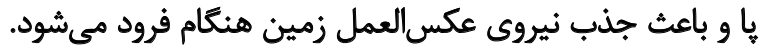

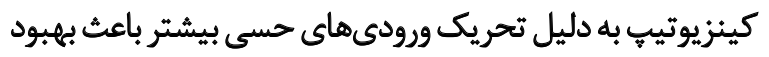

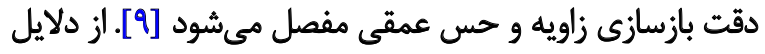

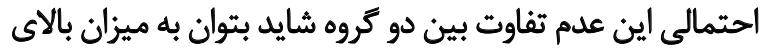




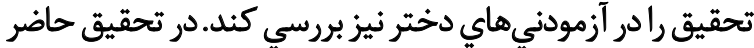

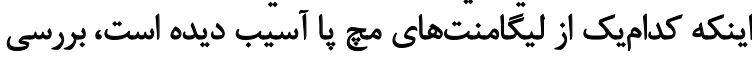

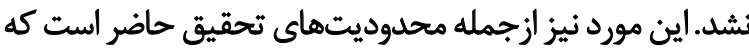
بهتر است در تحقيقات آينده بررسى شودئ

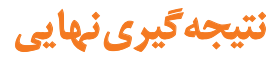

نتايج تحقيق حاضر نشان داد كه افراد دجار بي بيجخوردكى ميج

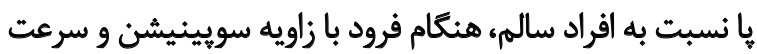

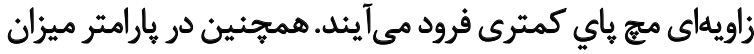

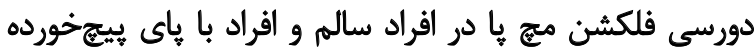

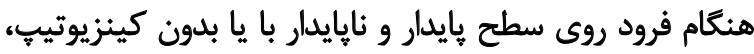

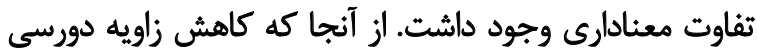

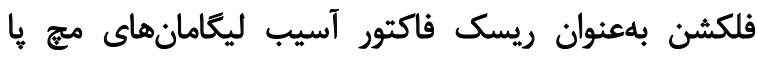

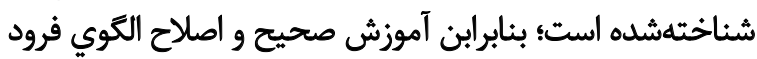

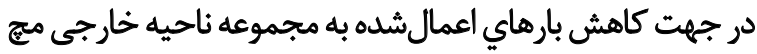

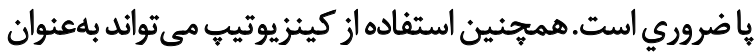
يك راه ييشكيرى از آسيب كمك كننده باشد.

مالاحظاث أخلاقي - اتي

\section{ييروى أز أصول انحلاق يؤوهش}

در اجراى يروهش ملاحظات اخلاقى مطابق با دستورالعمل

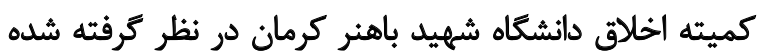

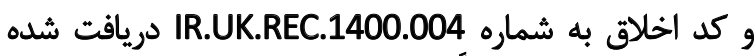

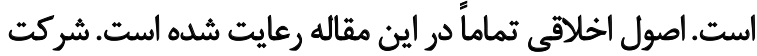

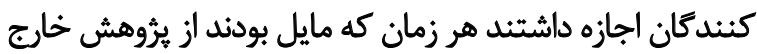

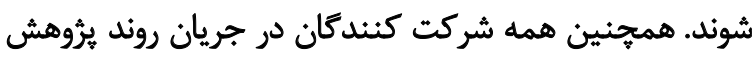

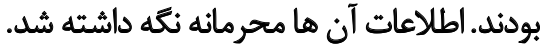

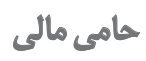

اين مقاله بركرفته از زايان نامه كارشناسي نويسنده اول در كروه

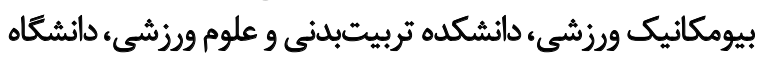
شهيد باهنر كرمان، كرمان است.

مشاركت ثويسندكًان

تمام نويسندكان در آمادهازى اين مقاله مشاركت يكسان

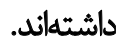

$$
\text { تعارض مثاقع }
$$

بنابر اظهار نويسندكان، اين مقاله تعارض منافع ندارد.

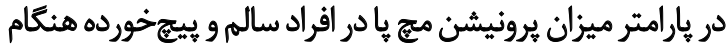

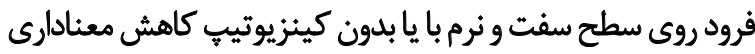

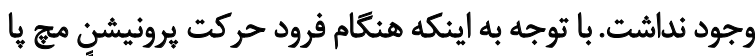

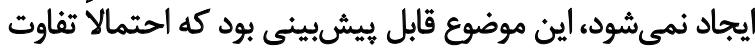

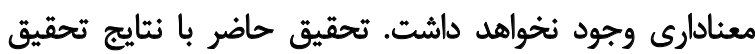

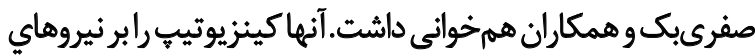

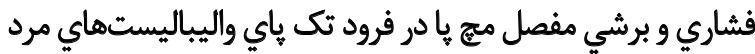

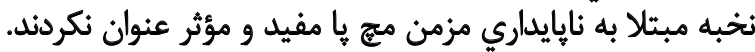

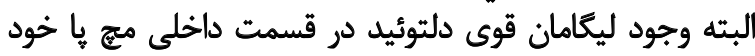

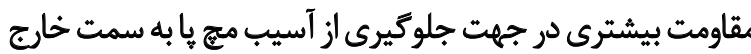

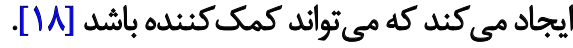

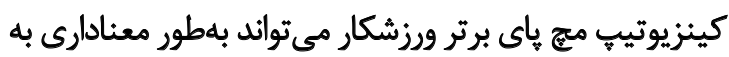

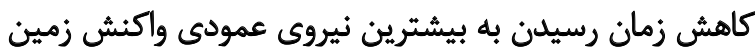

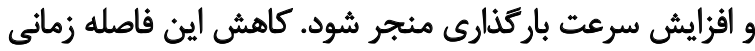

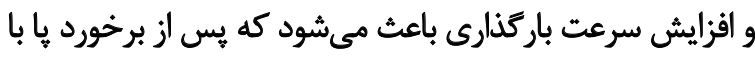

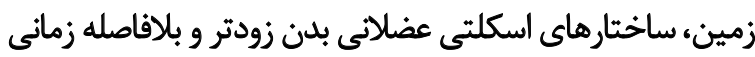

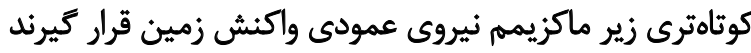

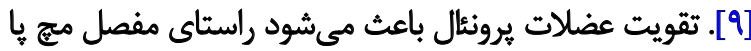

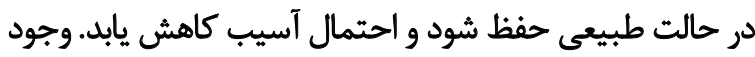

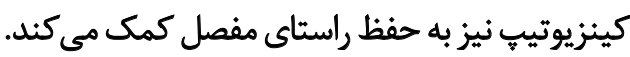

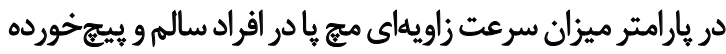

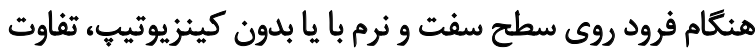

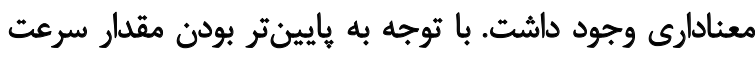

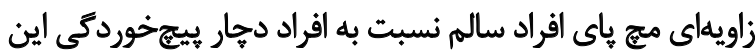

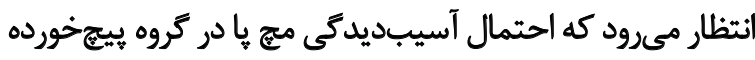

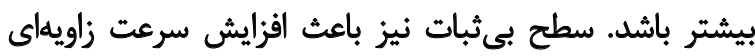

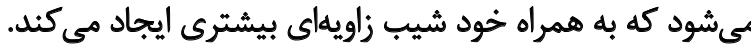

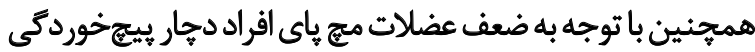

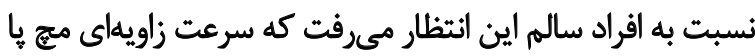

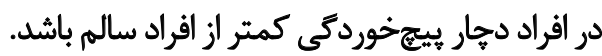

البته حضور كينزيوتيّ ميزان سرعت زاويهاى در دو گروه سالم

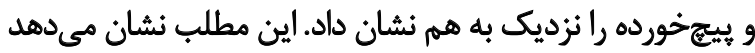

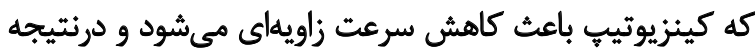

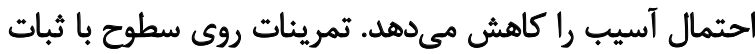

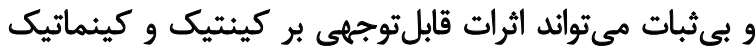

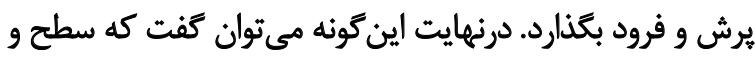

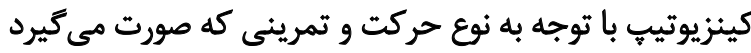

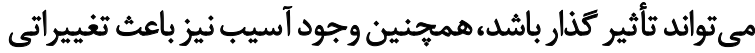
در ميزان بارامترهاي انتخابي مي شئشود.

محدوديتهاي تحقيق حاضر شامل بررسي روي دانشجويان

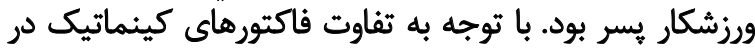

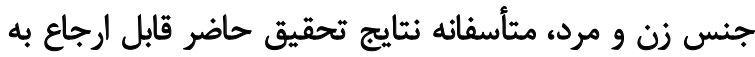

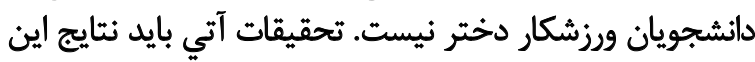




\section{References}

[1] McNair P, Prapavessis H, Callender K. Decreasing landing forces: Effect of instruction. British Journal of Sports Medicine 2000; 34(4):293-6. [DOI:10.1136/bjsm.34.4.293] [PMID] [PMCID]

[2] Dufek J, Bates B. Biomechanical factors associated with injury during landing in jump Sports. Sports Medicine. 1991; 12(5):326-37. [DOI:10.2165/00007256-199112050-00005] [PMID]

[3] Gray J, Taunton JE, McKenzie DC, Clement DB, McConkey JP, Davidson RG. A survey of injuries to the anterior cruciate ligament of the knee in female basketball players. International Journal of Sports Medicine. 1985; 6(6):314-6. [DOI:10.1055/s-2008-1025861] [PMID]

[4] Yeow CH, Lee PVS, Goh JCH. Sagittal knee joint kinematics and energetics in response to different landing heights and techniques. The Knee. 2010; 17(2):127-31. [DOI:10.1016/j. knee.2009.07.015] [PMID]

[5] Ferris DP, Farley CT. Interaction of leg stiffness and surface stiffness during human hopping. Journal of Applied Physiology. 1997; 82(1):15-22. [DOI:10.1152/jappl.1997.82.1.15] [PMID]

[6] Griffiths IW. Principles of biomechanics \& motion analysis. Philadelphia: Lippincott Williams \& Wilkins; 2006. https:// www.amazon.com/Principles-Biomechanics-Motion-AnalysisGriffiths/dp/0781762251

[7] Sadghi H, Abasi A, Khaleghi M. [Maximaal lower limb muscle torque and anterior knee shearing force during single leg landing (Persian)]. Research in Sports Science. 2007; 8(15):152-63. https://www.sid.ir/fa/journal/ViewPaper.aspx?id=159692

[8] Safari Bak M, Baharifard R, GandomKar A. [The effects of Kinesio tape on compressive and shear forces of ankle joint during single-leg landing for men elite volleyball players with chronic ankle instability (Persian)]. Research in Sports Medicine and Technology. 2021; 18(20):139-47. [DOI:10.29252/ jsmt.18.20.139]

[9] Botsis AE, Schwarz NA, Harper ME, Liu W, Rooney CA, Gurchiek LR, et al. Effect of kinesio ${ }^{\circledR}$ taping on ankle complex motion and stiffness and jump landing time to stabilization in female ballet dancers. Journal of Functional Morphology and Kinesiology. 2019; 4(2):19. [DOI:10.3390/jfmk4020019] [PMID] [PMCID]

[10] Lee S-M, Lee J-H. The immediate effects of ankle balance taping with kinesiology tape on ankle active range of motion and performance in the Balance Error Scoring System. Physical Therapy in Sport. 2017; 25:99-105. [DOI:10.1016/j. ptsp.2016.08.013] [PMID]

[11] Kim B-J, Lee JH, Kim CT, Lee SM. Effects of ankle balance taping with kinesiology tape for a patient with chronic ankle instability. Journal of Physical Therapy Science. 2015; 27(7):2405-6. [DOI:10.1589/jpts.27.2405] [PMID] [PMCID]

[12] Halim-Kertanegara S, Raymond J, Hiller CE, Kilbreath SL, Refshauge KM. The effect of ankle taping on functional performance in participants with functional ankle instability. Physical Therapy in Sport. 2017; 23:162-7. [DOI:10.1016/j. ptsp.2016.03.005] [PMID]
[13] Karimi-GhalehTal M, Akhbari B, Shaterzadeh MJ, Salavati M [Comparison ofankle taping effect on postural stability between fameles with functional ankle instability and healthy individuals (Persian)]. Archieve of Rehabilitation. 2007; 8(2):1723. https://rehabilitationj.uswr.ac.ir/article-1-160-en.htm

[14] Akhbari B, Takamjani IE, Salavati M, Sanjari MA. A 4-week biodex stability exercise program improved ankle musculature onset, peak latency and balance measures in functionally unstable ankles. Physical Therapy in Sport. 2007; 8(3):117-29. [DOI:10.1016/j.ptsp.2007.03.004]

[15] Robertson DG, Caldwell GE, Hamill J, Kamen G, Whittlesey S. Research methods in biomechanics. $2^{\text {th }}$ ed. Champaign: Human Kinetics; 2013. [DOI:10.5040/9781492595809]

[16] Little T, Williams AG. Effects of differential stretching protocols during warm-ups on high-speed motor capacities in professional soccer players. The Journal of Strength \& Conditioning Research. 2006; 20(1):203-307. [DOI:10.1519/R-16944.1] [PMID]

[17] Amiri-Khorasani M, MohammadKazemi R, Sarafrazi S, Riyahi-Malayeri $S$, Sotoodeh V. Kinematics analyses related to stretch-shortening cycle during soccer instep kicking after different acute stretching. The Journal of Strength \& Conditioning Research. 2012; 26(11):3010-7. [DOI:10.1519/ JSC.0b013e3182443442] [PMID]

[18] Hargrave MD, Carcia CR, Gansneder BM, Shultz SJ. Subtalar pronation does not influence impact forces or rate of loading during a single-leg landing. Journal of Athletic Training. 2003; 38(1):18-23. [PMCID]

[19] Halseth T, McChesney JW, DeBeliso M, Vaughn R, Lien J. The effects of kinesio ${ }^{\mathrm{TM}}$ taping on proprioception at the ankle. Journal of Sports Science \& Medicine. 2004; 3(1):1-7. [PMCID] [PMID]

[20] Niknam H, Sarmadi A, Salavati M, Madadi F. [The effect of knee kinesiotaping on proprioception and weight bearing in ACL reconstructed patients (Persian)]. Daneshvar Medicine. 2011; 19(2):33-42. http://daneshvarmed.shahed.ac.ir/article_3101.html?lang=en

[21] Shahidi-Zandi Z, Amir-seyfaddini MR, Amiri-Khorasani M. [Evaluation of lower extremity kinematic characteristics during single-leg landing from different heights in patients with knee valgus deformity (Persian)]. The Scientific Journal of Rehabilitation Medicine. 2017; 6(1):122-31. [DOI:10.22037/ JRM.2017.1100299]

[22] Yousefpour K, Shojaedin SS. [Determining the effectiveness of Kinesio Taping on balance in athletes with acute sprain and chronic ankles instable (Persian)]. Shahrekord University of Medical Sciences Journal . 2015; 17(5):83-93. https://www.sid. ir/en/journal/ViewPaper.aspx?ID=484616

[23] Robins S, Waked E, Rappel R. Ankle taping improves proprioception before and after exercise in young men. British Journal of Sports Medicine. 1995; 29(4):242-7. [DOI:10.1136/ bjsm.29.4.242]

[24] Mervat M, Lotfy RN, Ramadan AAS. Effect of Kinesio-taping on ankle joint stability. International Journal of Medical Research \& Health Sciences. 2016; 5(5):51-8. https://www.indianjournals.com/ijor.aspx?target=ijor:ijmrhs\&volume $=5 \&$ issue $=5$ \&article $=006$ 